\title{
Development and validation of a clinical index to predict survival after cardiac resynchronisation therapy
}

\author{
F Leyva, ${ }^{1}$ P W X Foley, ${ }^{1}$ B Stegemann, ${ }^{2}$ J A Ward, ${ }^{3} \mathrm{~L} \mathrm{~L} \mathrm{Ng},{ }^{4}$ M P Frenneaux, ${ }^{5} \mathrm{~F}$ Regoli, ${ }^{6}$ \\ R E A Smith, ${ }^{1}$ A Auricchio ${ }^{6}$
}

${ }^{1}$ University of Birmingham, Department of Cardiology, Good Hope Hospital, Heart of England NHS Trust, Sutton Coldfield, UK;

${ }^{2}$ Medtronic Inc, Bakken Research Center, Maastricht The Netherlands; ${ }^{3}$ University of Birmingham, Birmingham, UK;

${ }^{4}$ University of Leicester, Leicester, UK; ${ }^{5}$ Queen Elizabeth Hospital, University of Birmingham, Birmingham, UK;

${ }^{6}$ Fondazione Cardiocentro

Ticino, Lugano, Switzerland

Correspondence to:

Dr Francisco Leyva, Department of Cardiology, University of Birmingham, Good Hope Hospital, Rectory Road, Sutton Coldfield, West Midlands B75 7RR, UK;

cardiologists@hotmail.com

Accepted 23 June 2009 Published Online First 9 July 2009

\section{(6) UNLOCKF}

This paper is freely available online under the BMJ Journals unlocked scheme, see http:// heart.bmi.com/info/unlocked.dtl

\section{ABSTRACT}

Objective: To develop and validate a prognostic risk index of cardiovascular mortality after cardiac resynchronisation therapy (CRT).

Design: Prospective cohort study.

Setting: District general hospital.

Patients: 148 patients with heart failure (mean age 66.7 (SD 10.4) years), New York Heart Association class III or IV, LVEF <35\%) who underwent CRT.

Interventions: CRT device implantation.

Main outcome measures: Value of a composite index in predicting cardiovascular mortality, validated internally by bootstrapping. The predictive value of the index was compared to factors that are known to predict mortality in patients with heart failure.

Results: All patients underwent assessment of 16 prognostic risk factors, including cardiovascular magnetic resonance (CMR) measures of myocardial scarring (gadolinium-hyperenhancement) and dyssynchrony, before implantation. Clinical events were assessed after a median follow-up of 913 (interquartile range 967) days. At follow-up, 37/148 (25\%) of patients died from cardiovascular causes. In Cox proportional hazards analyses, (DSC) Dyssynchrony, posterolateral Scar location (both $p<0.0001)$ and Creatinine $(p=0.0046)$ emerged as independent predictors of cardiovascular mortality. The DSC index, derived from these variables combined, emerged as a powerful predictor of cardiovascular mortality. Compared to patients with a DSC $<3$, cardiovascular mortality in patients in the intermediate DSC index (3-5; HR: 11.1 (95\% confidence interval (CI) 3.00 to 41.1$), p=0.0003)$ and high DSC index ( $\geqslant 5$; HR: 30.5 (95\% Cl 9.15 to 101.8 ), $p<0.0001$ ) were higher. Bootstrap validation confirmed excellent calibration and internal validity of the prediction model.

Conclusion: The DSC index, derived from a standard CMR scan and plasma creatinine before implantation, is a powerful predictor of cardiovascular mortality after CRT.

The benefits of cardiac resynchronisation therapy (CRT) are well established. The Cardiac Resynchronisation in Heart Failure (CARE-HF) study showed that, compared to optimum medical therapy, CRT-pacing (CRT-P) led to a 36\% relative reduction in total mortality. ${ }^{1}$ The Comparison of Medical Therapy, Pacing and Defibrillation in Heart Failure (COMPANION) study showed that addition of a cardioverter defibrillator (CRT-D) led to higher survival benefits than CRT-P. ${ }^{2}$ Predicting which patients derive a survival benefit from CRT, however, remains a challenge. Numerous echocardiographic studies have focused on measures of cardiac dyssynchrony as predictors of outcome, but no consensus has been reached as to their clinical applicability. In the recent Predictors of Response to CRT (PROSPECT) study, which adopted left ventricular (LV) reverse remodelling as a surrogate of survival, tissue Doppler imagingbased measures of dyssynchrony were proved to add little value to ORS duration in predicting the outcome of CRT. ${ }^{4}$ No echocardiographic measure has been validated against mortality after CRT.

Cardiovascular magnetic resonance (CMR) is the gold standard for the in vivo assessment of myocardial structure, function and dyssynchrony. ${ }^{5}$ A recent pilot study has shown that a dyssynchrony measure derived from a standard CMR scan, the CMR tissue synchronisation index (CMR-TSI), is a powerful predictor of mortality and morbidity after CRT. ${ }^{6}$ As well as providing measures of dyssynchrony, CMR also allows visualisation and quantification of myocardial scarring with late gadolinium enhacement (CMRLGE)..$^{8}$ Importantly, the size (burden), transmurality and location of a myocardial scar in the LV free wall, the target location for LV leads, have been linked to a poor outcome after CRT. ${ }^{7-9}$

We have explored the value of pre-implant CMR measures of dyssynchrony and myocardial scarring, as well as more conventional heart failure risk factors, ${ }^{10}$ in predicting cardiovascular mortality after CRT. This study focuses on the derivation and validation of a clinical risk index based on a combination of such non-invasive measures.

\section{METHODS \\ Patients}

A total of 148 consecutive heart failure patients were prospectively enrolled from January 2001 to April 2008 at a single centre (Good Hope Hospital). Patients were selected if they were due to undergo CRT for standard indications (ischaemic or nonischaemic cardiomyopathy, New York Heart Association (NYHA) class III or IV, LV ejection fraction (LVEF) $<35 \%$, ORS duration $\geqslant 120 \mathrm{~ms}$ ). Until publication of the UK National Institute for Health and Clinical Excellence (NICE) Health Technology Appraisal in CRT in 2007, ${ }^{11}$ the implantation rate for CRT-D in the UK was very low. All patients included in this study underwent CRT-P.

All patients had undergone coronary angiography. The clinical diagnosis of systolic heart failure was based on clinical criteria and evidence of systolic dysfunction on echocardiography. The diagnosis of ischaemic cardiomyopathy was made 
if systolic dysfunction was associated with a history of myocardial infarction, ${ }^{12}$ the findings of coronary angiography, or if the pattern of LGE was characteristic of a myocardial infarction. ${ }^{13}$ All patients gave written informed consent and the study was approved by the North Birmingham ethics committee.

\section{Study design}

A CMR study was performed at baseline. The predictive risk index was validated against cardiovascular death. For qualitative comparisons with the CARE-HF study, we also considered the composite endpoint of death from any cause or hospitalisations for major cardiovascular events, as defined in the CARE-HF study protocol. ${ }^{1}$ Mortality data were collected through medical records and, where appropriate, from interviews with patients' caregivers. Clinical outcome data were collected on a twomonthly basis by an investigator who was blinded to other clinical and imaging data. Sudden cardiac death was defined as "a natural, unexpected death due to cardiac causes, heralded by an abrupt loss of consciousness within one hour of the onset of acute symptoms or occurring during the sleep without worsening of heart failure in the preceding hours." 14

\section{Device therapy}

Implantation of CRT-P devices was undertaken using standard techniques under local anaesthesia. The LV lead position was chosen by the implanter, who was blinded to the CMR study. Patients who underwent successful implantation were followed up in a dedicated device therapy clinic. Patients in sinus rhythm underwent trans-mitral Doppler-directed optimisation of atrioventricular delay ${ }^{15}$ before discharge and at every scheduled visit thereafter. For patients in atrial fibrillation, right ventricular and LV leads were implanted and a biventricular generator was used, plugging the atrial port and programming the generator to a ventricular-triggered mode. All patients were proved to have functional leads and generators throughout the study period.

\section{Cardiovascular magnetic resonance}

Imaging was performed using a 1.5 Tesla scanner (Signa, GE Healthcare, Slough, UK) and a phased array cardiac coil. A short axis LV stack was acquired using a steady state in free precession sequence (SSFP, repetition time 3.0-3.8 ms; excitation time $1.0 \mathrm{~ms}$; image matrix $224 \times 224$; field of view $36-$ $42 \mathrm{~cm}$; flip angle $45^{\circ}$ ) in sequential $8-\mathrm{mm}$ slices (2-mm interslice gap) from the atrio-ventricular ring to apex. Acquisition was performed during gated 8-second breath-holds. Left ventricular end diastolic volume (LVEDV) and end-systolic (LVESV) volume, and LVEF were quantified using manual planimetry of all short-axis SSFP cine images with MASS analysis software (Medis, Leiden, The Netherlands). The observer was blinded to echocardiographic and clinical data.

The CMR-TSI was measured as previously described. ${ }^{6}$ Briefly, the maximum radial wall motion value of a radial wall motion time series was chosen as the peak radial wall motion for each LV segment. The time-dependent segmental radial wall motion data $(y)$ were fitted to an empirical sine wave function $y=a+$ $b \times \sin (t / R R+c)$. The mean segmental radial wall motion (a), the segmental radial wall motion amplitude (b) and the segmental phase shift of the maximum radial wall motion (c) were extracted from the fit. The non-linear least squares fit function implemented by Bates and DebRoy into the "nls" packet of $\mathrm{R}$ was used for the sine fit. ${ }^{16}$ The CMR-TSI was expressed as the SD of all segmental phase shifts of the radial wall motion extracted from the fit. Therefore, the CMR-TSI is a measure of the temporal dispersion of peak inward myocardial motion throughout the cardiac cycle. A high CMR-TSI, therefore, denotes high temporal dispersion of inward wall motion.

For scar imaging using CMR-LGE, short-axis slices identical to the LV stack were acquired using a segmented inversionrecovery technique 10 minutes after the intravenous administration of gadolinium-diethylenetriamine pentaacetic acid $(0.1 \mathrm{mmol} / \mathrm{kg})$. Inversion times were adjusted to null normal myocardium (260-400 ms). Scar volume was calculated by multiplying the planimetered area of LGE in each slice by the slice thickness. Scar volume was expressed as a percentage of LV myocardial volume in the diastolic phase. Areas of scar were then segmented using a 17 -segment model. ${ }^{17}$ A posterolateral scar was defined as a scar which, in this model, was located in segments $6,12,16,5$ or 11 , or in the lateral half of segments 1,7 , $13,17,15,10$ and 4. Transmurality was also assessed using a 17segment model. ${ }^{17}$ A transmural posterolateral scar was defined as a scar with $51-100 \%$ of the LV wall, measured radially.

\section{Statistical analysis}

Continuous variables are expressed as mean (SD). Normality was tested using the Shapiro-Wilk test. Variables which were not normally distributed were log-transformed before statistical analyses. Comparisons between normally distributed continuous variables were made using ANOVA with Scheffe's F procedure for multiple comparisons. Categorical variables were analysed using $\chi^{2}$ tests and Scheffe's post-hoc test. Differences in Kaplan-Meier survival curves between the risk groups were assessed using the log-rank (Mantel-Cox) test. Areas under the receiver-operating characteristic (ROC) curves (AUC) were used to derive optimal cut-off points for the DSC index. Statistical analyses were performed using Statview and SPSS 13.0. Harrel's "Design" package version 2.1-1in R was used for predictive modelling. ${ }^{18} \mathrm{~A}$ two-tailed $\mathrm{p}$ value of $<0.05$ was considered statistically significant. In a previous study, ${ }^{6}$ intra-observer and inter-observer variabilities for CMR-TSI were $3.0 \%$ and $8.8 \%$, respectively.

\section{Development of the risk index}

In line with Harrell's recommendations on multivariate prognostic modelling, ${ }^{192}$ no more than $m / 10$ parameters were considered, where $m$ is the number of uncensored events, in this case cardiovascular deaths $(n=37)$. Candidate variables were selected on the basis of their proved clinical, pathophysiological and epidemiological relevance to the endpoint in question. A measure of cardiac dyssynchrony-namely, CMT-TSI, was included in view of previous demonstration from echocardiographic $^{21}$ and radionuclide ${ }^{22}$ studies that extremes of dyssynchrony relate to a poor outcome in patients with heart failure. Similarly, a posterolateral location of myocardial scar was chosen in view of its negative influence on outcome after CRT. ${ }^{9}$ Plasma creatinine was also selected given its emergence as a predictor of mortality in patients in heart failure in numerous studies. ${ }^{10}{ }^{23-27}$ These three variables were included in the multiple regression model. Continuous rather than dichotomous variables were used wherever possible. ${ }^{20}$ Interactions between variables were extensively explored using interaction terms in Cox proportional hazards models. Linearity assumptions were checked using Martingale residuals. Cross-product terms were added to check for additivity assumptions. The proportional hazards assumption was checked using Schoenfeld residuals. ${ }^{28}$ 
Table 1 Clinical characteristics of the study group

\begin{tabular}{|c|c|}
\hline Characteristics & $\mathrm{n}=148$ \\
\hline Age (years) & $66.7(10.4)$ \\
\hline Men (\% of patients) & $114(77)$ \\
\hline Ischaemic aetiology (\% of patients) & $92(62)$ \\
\hline NYHA class & $3.2(0.43)$ \\
\hline Creatinine $(\mu \mathrm{mol} / \mathrm{l})$ & $119.8(38.3)$ \\
\hline Sodium (mmol/l) & $139.0(5.8)$ \\
\hline Haemoglobin (g/dl) & $14.1(7.9)$ \\
\hline \multicolumn{2}{|l|}{ Co-morbidity, No (\% of patients) } \\
\hline Diabetes mellitus & $24(16)$ \\
\hline Hypertension & $40(27)$ \\
\hline CABG & $31(21)$ \\
\hline \multicolumn{2}{|l|}{ Medication, No (\% of patients) } \\
\hline Loop diuretics & $129(87)$ \\
\hline ACE-I or ARB & $136(92)$ \\
\hline$\beta$-blockers & $81(55)$ \\
\hline Spironolactone & $66(45)$ \\
\hline \multicolumn{2}{|l|}{ ECG variables } \\
\hline Atrial fibrillation, № (\% of patients) & $23(16)$ \\
\hline ORS duration (ms) & $145.9(26.5)$ \\
\hline \multicolumn{2}{|l|}{ CMR variables } \\
\hline LVEDV (ml) & 247.8 (99.5) \\
\hline LVESV (ml) & 200.2 (96.6) \\
\hline $\operatorname{LVEF}(\%)$ & $22.5(10.6)$ \\
\hline CMR-TSI (ms) & $100.7(47.8)$ \\
\hline \multicolumn{2}{|l|}{ Scar* } \\
\hline Volume (\% of LV myocardial volume) & $19.5(20.1)$ \\
\hline Transmural, № (\% of patients) & $62(42)$ \\
\hline Posterolateral, No (\% of patients) & $48(32)$ \\
\hline \multicolumn{2}{|c|}{$\begin{array}{l}\text { Continuous variables are expressed as mean (SD). } \\
{ }^{*} \text { Transmural scars were those with } \geqslant 51 \% \text { transmurality. } \\
\text { ACE-I, angiotensin-converting enzyme inhibitors; ARB, angiotensin- } \\
\text { receptor blockers; CABG, coronary artery bypass graft; CMR-TSI, } \\
\text { cardiovascular magnetic resonance tissue synchronisation index; } \\
\text { LVEDV, left ventricular end-diastolic volume; LVEF, left ventricular } \\
\text { ejection fraction; LVESV, left ventricular end-systolic volume; NYHA, } \\
\text { New York Heart Association. }\end{array}$} \\
\hline
\end{tabular}

\section{Validation}

Validation was implemented using bootstrapping. ${ }^{19} 2029$ This non-parametric method, which estimates sampling distributions of a predictor variable by resampling with replacement from the original sample, is, in effect, an impression of the validity of predictions in new but similar patients. For each group of 200 bootstrap samples, the model was refitted and tested against the observed sample in order to derive an estimate of the predictive accuracy and bias. This was used to select 148 "new" patients from the original cohort, one at a time with replacement, 200 times over. For each of these 200 samples, the following parameters were derived: (1) the $\beta$ coefficients from Cox proportional hazards models; (2) Somer's D rank correlation index and (3) an estimate of the slope shrinkage. ${ }^{19}$ The apparent Somer's D ( $\left.\mathrm{D}_{\text {app }}\right)$ was derived using stepwise Cox proportional analyses. The bootstrap-corrected performance of the predictor equation, or "optimism", ${ }^{20}$ was quantified by assessing the difference between Somer's D in the original sample $\left(D_{\text {orig }}\right)$ and $D$ in the bootstrap sample $\left(D_{\text {boot }}\right)$. The average optimism, termed $\mathrm{O}$, was derived by repeating the above steps 200 times over. The bootstrap-corrected performance of the original stepwise model, $\mathrm{D}_{\mathrm{app}}-\mathrm{O}$, is, effectively, an honest estimate of internal validity. ${ }^{20}$ Actual survival was plotted against apparent estimated survival and bootstrapcorrected estimated survival.

\section{Calculation of the risk index}

The risk prediction index was expressed as the absolute value of the sum of the products of these variables and their $\beta$ coefficients (that is, weights) from the final stepwise multivariate Cox proportional hazards model, identified by Harrels's routine ${ }^{19}{ }^{20}: \Sigma \beta_{\mathrm{i}} x_{\mathrm{i}}=\left|\beta_{1} x_{1}+\beta_{2} x_{2}+\ldots+\beta_{\mathrm{n}} x_{\mathrm{n}}\right|$, where $x_{1}, x_{2}, \ldots x_{\mathrm{n}}$ are the values for the variables and $\beta_{1}, \beta_{2}, \ldots \beta_{\mathrm{n}}$ are the coefficients for each variable. For categorical variables, presence was computed as the $\beta$ coefficient and absence as zero.

\section{Univariate analyses}

Univariate Cox proportional hazards analyses were performed after development and validation of the DSC index. These were undertaken in order to assess the relative strength of other variables in relation to cardiovascular mortality.

\section{RESULTS}

The characteristics of the study group are shown in table 1 . There were 20 cardiovascular deaths by one year and 28 by two years. After a follow-up of 913 (967) days (median (interquartile range) for events, there were 37 cardiovascular deaths. Of these, 28 were due to pump failure, eight were sudden and one patient underwent cardiac transplantation. Of the 48 patients with a posterolateral scar, 35 had a transmural scar and 12 had a nontransmural scar. Scar volume in these 48 patients was $33.2 \%$ (18.5).

\section{Derivation and validation}

Table 2 shows model outputs for the variables included in the prediction model (DSC): Dyssynchrony, Scar location (posterolateral) and Creatinine. The DSC index was calculated as follows: (2.5039 if posterolateral scar present; 0 if absent) + 0.0107. CMR-TSI $(\mathrm{ms}))+(0.0132$. plasma creatinine $(\mu \mathrm{mol} / \mathrm{l}))$.

Strata of the DSC index were designated as low, intermediate or high risk, with cut-offs of $<3$, between 3 and 5 and $\geqslant 5$. These were chosen arbitrarily for ease of use in clinical practice. Kaplan-Meier analyses were used to assess survival according strata of the DSC index (fig 1). Among the statistics derived from Harrell's routine ${ }^{18} 20$ was a bias-corrected slope of the

Table 2 Final model from bootstrapped multivariable Cox proportional hazard analyses of predictors of mortality

\begin{tabular}{|c|c|c|c|c|}
\hline & $\beta$ coefficient $(95 \% \mathrm{CI})$ & HR $(95 \%$ CI) & Z-score & p Value \\
\hline $\begin{array}{l}\text { Posterolateral scar } \\
\text { location }\end{array}$ & 2.50 (1.60 to 3.40$)$ & 12.2 (4.97 to 30.1$)$ & 5.46 & $<0.0001$ \\
\hline CMR-TSI $(\mathrm{ms})^{*}$ & $0.01(0.00$ to 0.02$)$ & $1.01(1.00$ to 1.02$)$ & 3.26 & 0.0011 \\
\hline Creatinine $(\mu \mathrm{mol} / \mathrm{l})$ & $0.01(0.00$ to 0.02$)$ & $1.01(1.00$ to 1.02$)$ & 2.83 & 0.0046 \\
\hline
\end{tabular}

${ }^{*}$ Consequently a CMR-TSI of $100 \mathrm{~ms}$ is associated with a hazard ratio of 2.

$\mathrm{Cl}$, confidence interval; CMR-TSI, cardiovascular magnetic resonance tissue synchronisation index; HR, hazard ratio. 
Figure 1 Kaplan-Meier estimates of the time to cardiovascular death. Patients were stratified according to pre-implant DSC index. The event rate, number of patients in the DSC risk stratum and the percentage event rate are shown in brackets. The hazard ratio (HR) and 95\% confidence intervals are also shown.

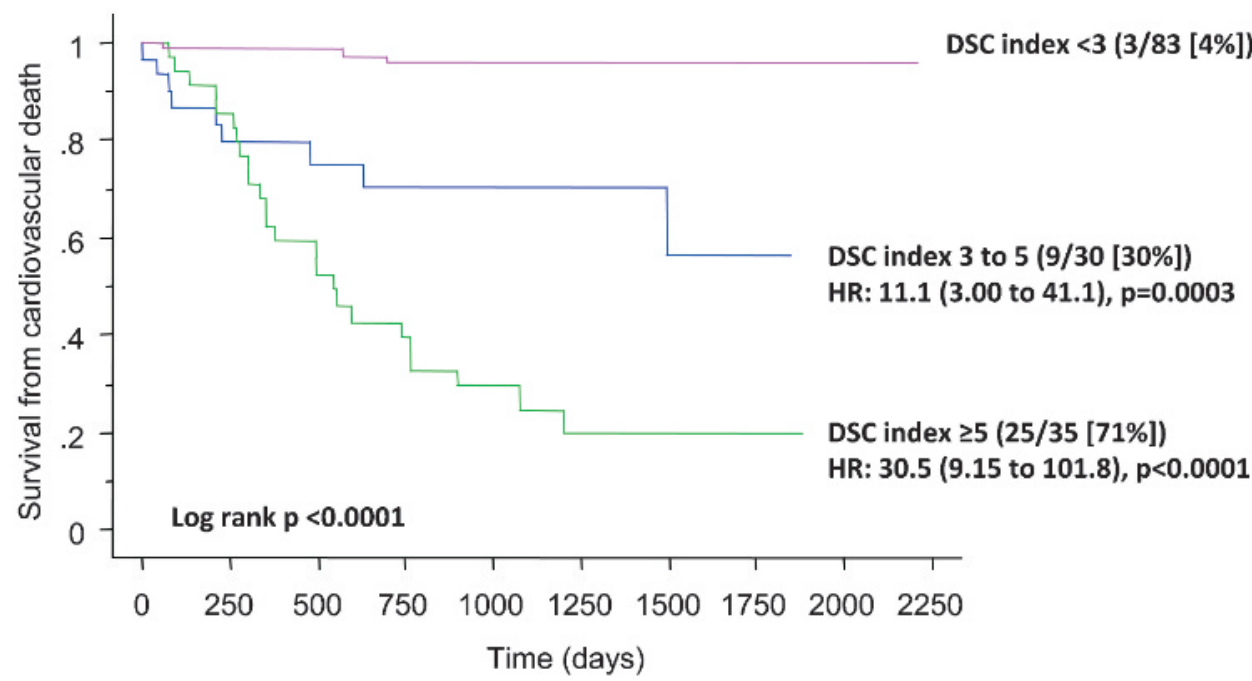

model of 0.93 , indicating an excellent model calibration, taking into account overfitting (fig 2). Somer's $\mathrm{D}_{\text {app was }}-0.70$ and the optimism $(\mathrm{O})$ was -0.01 . The bias-corrected performance of the stepwise model $\left(\mathrm{D}_{\mathrm{app}}-\mathrm{O}\right)$ was acceptable, at -0.69 , indicating excellent internal validity. The optimism-corrected discrimination index was 0.91 ; the optimism-corrected unreliability index $\mathrm{U}$ was 0.01 and the overall quality index was 0.85 . The ROC for the DSC index in relation to cardiovascular mortality at various time points is shown in figure 3.

\section{Univariate analyses}

Table 3 shows univariate model outputs for other candidate predictors, ranked according to the likelihood ratio (LR) $\chi^{2}$. Among the imaging variables, posterolateral scar location (LR $\chi^{2}$ : 58.6) and CMR-TSI (LR $\left.\chi^{2}: 25.5\right)$ emerged as the most significant predictors (all $\mathrm{p}<0.0001)$. Scar burden (LR $\chi^{2}: 13.3$ ) and CMR-LVEF (LR $\left.\chi^{2}: 11.1\right)$ were also strong predictors (both $\mathrm{p}<0.001$ ). Among circulating markers, sodium and creatinine were significant predictors (both $\mathrm{p}<0.01$ ), uric acid only reached borderline significance $(p=0.0808)$ and haemoglobin failed to

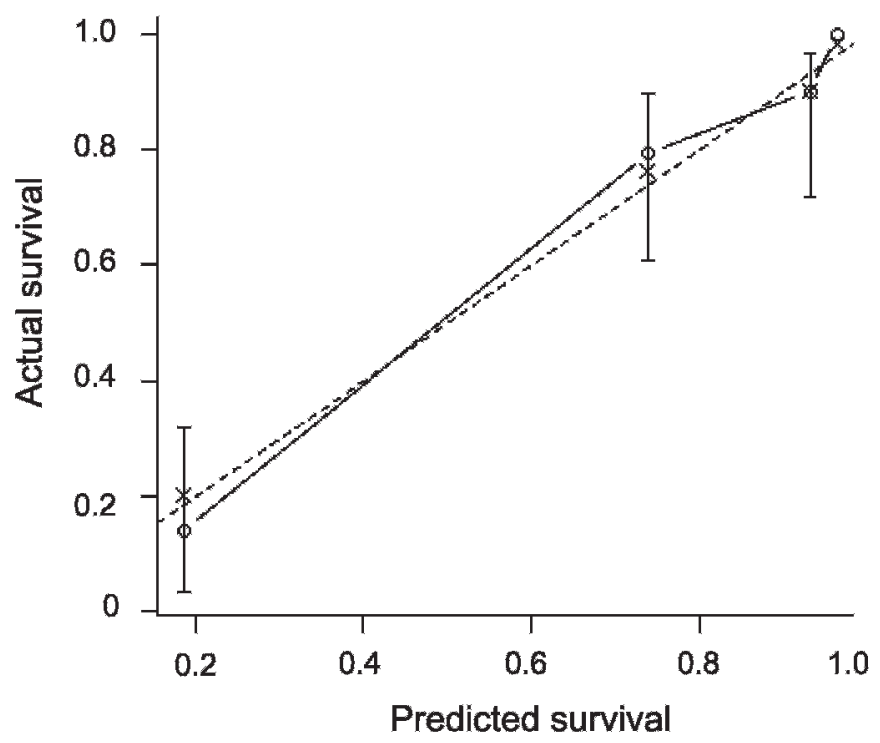

Figure 2 Calibration of predictions of mortality after cardiac resynchronisation therapy. Graph shows actual versus bootstrapcorrected predicted survival. reach significance. Male gender was also a modest predictor $(p=0.0417)$. Age, NYHA class, ORS duration, atrial fibrillation, diabetes or CABG failed to reach statistical significance. Similarly, use of loop diuretics, angiotensin-converting enzyme inhibitors (ACE)/angiotensin-receptor blockers, $\beta$-blockers or spirinolactone failed to emerge as predictors of cardiovascular mortality (data not shown). Although ischaemic aetiology did emerge as a significant predictor of cardiovascular mortality in univariate analysis, addition of scar burden to this model rendered aetiology nonsignificant (data not shown). The decision not to include ischaemic aetiology in the multiple regression model was taken in view of its expected interaction with scar burden and scar location (non-ischaemic cardiomyopathy is associated with little or no scar). In addition, the diagnoses of ischaemic and nonischaemic cardiomyopathy were made on the basis of size, transmurality and location of scar in the CMR scan.

\section{DISCUSSION}

Our findings have emerged in the context that there are, hitherto, no means of predicting mortality in patients undergoing CRT. Our aim was to identify an index that could predict cardiovascular mortality after CRT on the basis of pre-implant variables. The DSC index, which combines pre-implant measures of dyssynchrony and location of myocardial scar as well as creatinine, is a novel, powerful predictor of cardiovascular mortality in patients treated with CRT. Compared to patients in the lowest risk stratum, cardiovascular mortality values in the intermediate and high-risk strata of the DSC index were 11.1 and 30.5 times higher by the end of the follow-up period, respectively.

The variables from which the DSC is derived have previously been linked to a poor outcome after CRT. Of particular mechanistic relevance to CRT is scar burden and scar location. This study confirms the findings of previous studies showing that the presence of myocardial scar over the posterolateral LV wall is a powerful predictor of cardiovascular mortality. ${ }^{8}$ The precise pathophysiological mechanism is still unclear, but several factors may contribute to this observation. First, myocardial scars are not readily excitable. ${ }^{30}$ A posterolateral scar reduces the volume of excitable myocardium in the vicinity of the LV pacing stimulus and delays activation of potentially recruitable myocardium, which is crucial for CRT. ${ }^{31}$ Second, a posterolateral scar also reduces the amount of myocardium available for contraction in CRT, thereby leading to suboptimal 


\section{1 year}

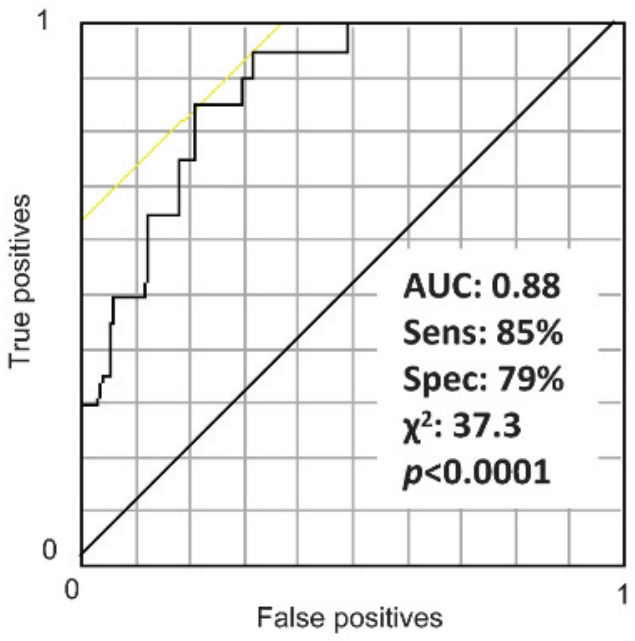

2 years

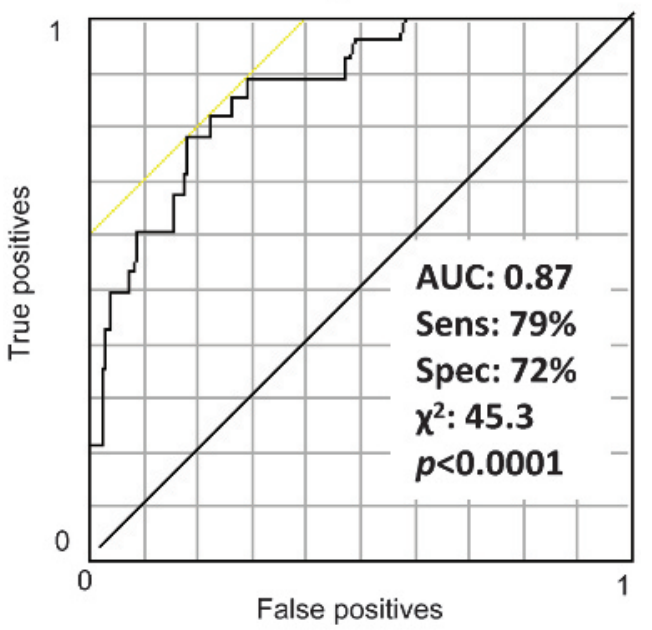

Total follow-up period

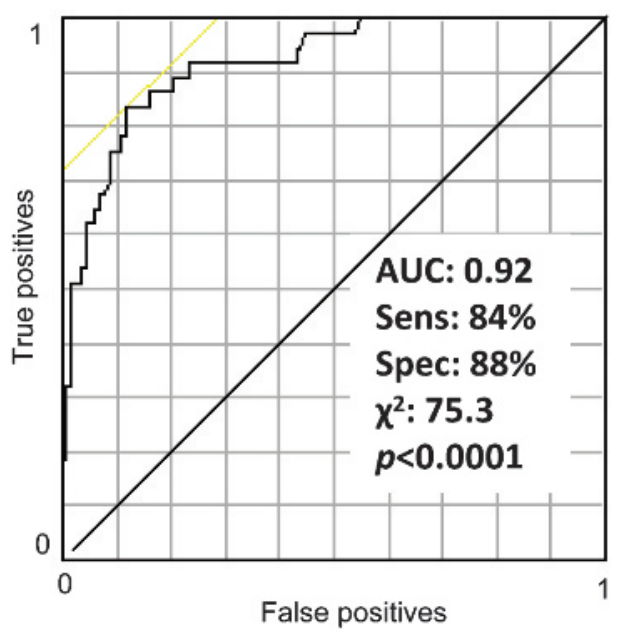

Figure 3 Receiver-operator characteristic curves of the DSC index against cardiovascular mortality at 1 and 2 years, and at the end of the follow-up period. AUC, area under the curve; sens, sensitivity, spec, specificity. Values refer to a DSC cut-off of 4.7. reverse LV remodelling following CRT. ${ }^{32}$ Third, posterolateral scarring may also preclude proper papillary muscle function, which is essential for competence of the mitral valve.

As in a previous study, ${ }^{6}$ we have shown that dyssynchrony, assessed using CMR-TSI, predicts mortality after CRT. ${ }^{6}$ These findings are in contrast to numerous echocardiographic studies showing that increasing dyssynchrony relates to increasing benefit. In other words, our findings point towards a "too much dyssnchrony is bad" paradigm, whereas echocardiographic studies support an "any degree of dyssynchrony is good" paradigm of CRT. Our finding that "too much dyssynchrony is bad" is, however, consistent with the finding that in non-paced patients with heart failure, dyssynchrony, assessed by tissue Doppler imaging, is a predictor of cardiac decompensation. ${ }^{21} \mathrm{~A}$ radionuclide radioscintigraphy study of patients with dilated cardiomyopathy has also shown that a dyssynchrony index was an independent predictor of major cardiac events, including cardiovascular death. ${ }^{22}$ Moreover, Bax et al ${ }^{33}$ recently found that beyond a limit of dyssynchrony (a septal-to-posterior wall motion delay of $100 \mathrm{~ms}$ ), CRT does not result in reverse LV remodelling. The "too much dyssynchrony is bad" paradigm might be expected in view that dyssynchrony is inversely related to LVEF, ${ }^{6}$ and positively related to LV volumes ${ }^{6}$ and ORS duration. ${ }^{6435}$ Together, these data suggest that the most damaged and worst functioning left ventricles gain the least benefit from CRT. In other words, that there is a maximum of treatable dysynchrony.

Our findings are in contrast to those of the PROSPECT study, in which echocardiographic measures of dyssynchrony were shown to be of little additive value to ORS duration in predicting functional and LV volumetric changes after CRT. ${ }^{4}$ Prominent among the findings of this study were the very high coefficients of variation for echocardiographic dyssynchrony measures, which were as high as $72 \%{ }^{4}$ Current technology, degree of training standards and analytical methods do not allow incorporation of echocardiographic measures of dyssynchrony in a generalised setting, representing a significant limitation for their widespread use. ${ }^{36}$ In contrast, the intraobserver and inter-observer variabilities for CMR-TSI are as low as $3.01 \%$ and $8.84 \%$, respectively, ${ }^{6}$ thereby providing the highest accuracy and lowest intra-observer and inter-observer variabilities for a dyssynchrony measure.

We have also considered other non-invasive variables which have previously been shown to be of prognostic value in patients with heart failure treated ${ }^{7}$ and not treated ${ }^{102627}$ with CRT. The emergence of plasma creatinine in the predictive model is not surprising because renal impairment is a predictor of poor outcome in non-paced patients with heart failure. ${ }^{23-26}{ }^{37}$ We have shown that the inverse relation between plasma creatinine and survival still holds in patients with heart failure undergoing CRT.

\section{Clinical application}

Cardiovascular magnetic resonance imaging is becoming a key investigation in patients with cardiomyopathy, as it provides information on aetiology, ventricular function and prognosis. ${ }^{13}{ }^{38}$ Myocardial tagging, ${ }^{39} 40$ strain-coding ${ }^{5}$ and velocityencoding ${ }^{41}$ techniques are all capable of providing dyssynchrony measures. These techniques, however, are highly specialised, are not routinely available in any centre and, more importantly, have not been validated against mortality after CRT. In contrast, the CMR technique that allows derivation of the DSC index involves a routine CMR-LGE scan, which is available in any CMR centre. With the emergence of software packages that allow rapid assessment of myocardial scars and wall motion, the DSC index can be easily quantified. 
Table 3 Univariate Cox proportional hazard analyses of predictors of mortality

\begin{tabular}{|c|c|c|c|c|}
\hline & $\beta$ coefficient $(95 \%$ Cl) & HR (95\% Cl) & $\operatorname{LR} \chi^{2}$ & p Value \\
\hline Posterolateral scar location & $2.82(1.94$ to 3.70 & $16.8(6.94$ to 40.5$)$ & 58.6 & $<0.0001$ \\
\hline CMR-TSI (ms) & $0.015(0.01$ to 0.02$)$ & 1.01 (1.01 to 1.02$)$ & 25.5 & $<0.0001$ \\
\hline Scar burden (\%) & $0.03(0.01$ to 0.04$)$ & $1.03(1.01$ to 1.04$)$ & 13.3 & 0.0001 \\
\hline CMR-LVEF (\%) & $-0.06(-0.10$ to -0.02$)$ & $0.94(0.90$ to 0.98$)$ & 11.1 & 0.0032 \\
\hline Sodium (mmol/l) & $-0.13(0.04$ to -0.04$)$ & $0.88(0.81$ to 0.96$)$ & 8.2 & 0.0031 \\
\hline Creatinine $(\mu \mathrm{mol} / \mathrm{l})$ & 0.01 (0.00 to 0.02 ) & $1.01(1.00$ to 1.02$)$ & 7.92 & 0.0049 \\
\hline Transmurality (transmural) & $0.92(0.26$ to 1.58$)$ & 2.51 (1.29 to 4.84$)$ & 7.64 & 0.0057 \\
\hline Male gender & $0.95(-0.08$ to 1.99$)$ & $2.59(0.92$ to 7.32$)$ & 4.15 & 0.0417 \\
\hline Uric acid $(\mu \mathrm{mol} / \mathrm{l})$ & $0.00(-0.00$ to 0.00$)$ & $1.00(1.00$ to 1.00$)$ & 2.33 & 0.1265 \\
\hline NYHA class & $0.54(-0.17$ to 1.21$)$ & $1.73(0.84$ to 3.37$)$ & 2.24 & 0.1343 \\
\hline CABG & -0.28 (0.18 to -0.62$)$ & $0.75(0.53$ to 1.08$)$ & 2.42 & 0.1196 \\
\hline QRS duration (ms) & $0.01(-0.00$ to 0.02$)$ & 1.01 (1.00 to 1.02$)$ & 1.01 & 0.2202 \\
\hline Age (years) & $0.01(0.02$ to -0.01$)$ & 1.01 (0.98 to 1.05 ) & 0.78 & 0.3765 \\
\hline Atrial fibrillation & $0.15(-0.23$ to 0.49$)$ & $1.16(0.80$ to 1.63$)$ & 0.64 & 0.4220 \\
\hline Diabetes mellitus & $-0.17(-0.55$ to 0.28$)$ & $0.84(0.57$ to 1.33$)$ & 0.61 & 0.4324 \\
\hline Haemoglobin (g/dl) & $-0.02(-0.19$ to 0.02$)$ & 0.98 (0.83 to 1.02 ) & 0.51 & 0.4763 \\
\hline
\end{tabular}

Variables are listed in order of statistical significance and likelihood ratio $\chi^{2}\left(\mathrm{LR} \chi^{2}\right)$.

$\mathrm{CABG}$, coronary artery bypass graft; $\mathrm{Cl}$, confidence interval; CMR-TSI, cardiovascular magnetic resonance tissue synchronisation index; CMR-LVEF, cardiovascular magnetic resonance left ventricular ejection fraction; HR, hazard ratio; NYHA, New York Heart Association.

\section{Limitations of the study}

All patients in this study underwent CRT-P. Therefore, the DSC index may not be generalisable to a population of patients treated with CRT-D. External validation of the DSC index in a cohort of patients undergoing CRT-D would be desirable. As a further limitation, the use of $\beta$-blockers and spironolactone were relatively low. While drug therapy failed to emerge as a predictor of cardiovascular mortality, we cannot discount the possibility that the relation between the DSC index and cardiovascular mortality might be influenced by more optimised medical therapy. On the other hand, in prospective randomised controlled trials, absence of $\beta$-blocking agents or spironolactone was not associated with a significant survival benefit. ${ }^{1}$

The DSC index may be useful in predicting the outcome of CRT, but a high DSC index should not be used to deny CRT to patients who satisfy current guideline criteria. As our study did not include a control group, one cannot be certain the CRT-P led to a prognostic benefit. This, however, seems likely in view of the similarity between the prognostic risk profile of the present cohort and that of the CRT-P arm of the CARE-HF study (fig 4).

\section{CONCLUSIONS}

This study comprised patients with heart failure in NYHA class III and IV, such as those included in the CARE-HF study. We have found that in such patients, the DSC index is a powerful riskstratifier for cardiovascular mortality. Patients in the highest risk stratum were more than 30 times as likely to die from cardiovascular causes than those in the low risk stratum. These findings are perhaps not surprising, as extreme dyssynchrony, a posterolateral location of scar and poor renal function have all been linked to a poor outcome in patients with heart failure. The present study supports the use of a standard CMR-LGE in prospective CRT candidates, in whom pre-implantation risk stratification may influence clinical management. Although this study did not include patients undergoing CRT-D, it seems unlikely that patients in the highest DSC risk category, most of whom die of pump failure, would derive a survival benefit from CRT-D. On this basis, the DSC index may influence resource allocation.
Figure 4 Comparison of events in the present cohort and in the CARE-HF study. Qualitative comparison of Kaplan-Meier survival estimates for the combined endpoint of death from any cause or hospitalisations for major cardiovascular events (MCE) for patients included in this study as well as those in the CRT-P and the optimum medical treatment (OPT) arms the CARE-HF study. Follow-up time in the present cohort has been truncated to 1500 days. Modified with permission from Cleland et al.'

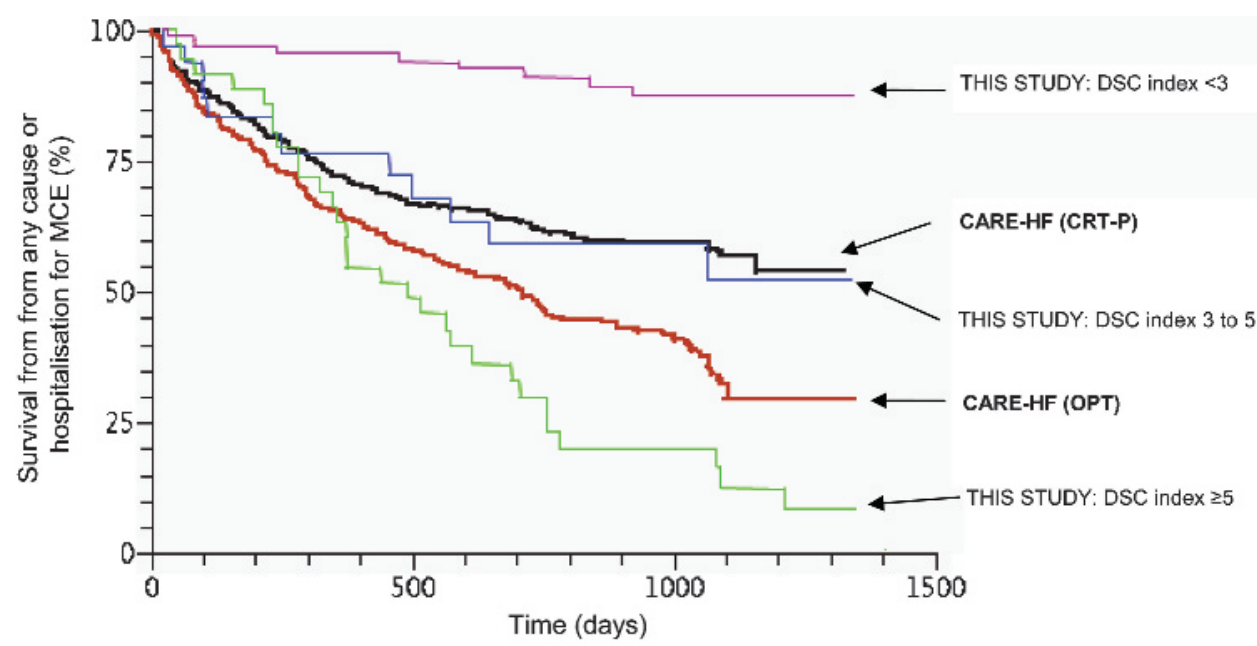


Acknowledgements: We are grateful to Professor Nick Freemantle for statistical advice. We are also grateful to Lisa Ball, Janet Brashaw-Smith and Nick Irwin for their input in the follow-up of patients included in this study.

Funding: The role of the industry sponsors was, exclusively, to fund research fellowships. BS, who was employed by Medtronic Inc, at the time of writing, provided statistical expertise and was involved in the review of the manuscript. The other authors had full independence from the sponsors with regard to study design, data collection, interpretation and manuscript preparation.

Competing interests: SC and PF have research fellowships sponsored by Medtronic Inc. BS was an employee of Medtronic Inc. REAS, PJ and FL have received sponsorship from Medtronic Inc. FL and KK have also received sponsorship from St Jude Medical. MPF is a consultant for Medtronic Inc. AA has research fellowships sponsored by Medtronic Inc, Boston Scientific Corp and St Jude Medical, and is consultant for Medtronic Inc, and Sorin. He has also received speaker fees from Medtronic Inc, Sorin and Biotronik.

Provenance and peer review: Not commissioned; externally peer reviewed.

\section{REFERENCES}

1. Cleland JGF, Daubert J-C, Erdmann E, et al, for the Cardiac Resynchronization-Heart Failure (CARE-HF) study investigators. The effect of cardiac resynchronization on morbidity and mortality in heart failure. N Engl J Med 2005;352:1539-49.

2. Bristow MR, Saxon LA, Boehmer J, et al, for the Comparison of Medical Therapy, Pacing and Defibrillation in Heart Failure (COMPANION) Investigators. Cardiac resynchronization therapy with or without an implantable defibrillator in advanced heart failure. N Engl J Med 2004;350:2140-50.

3. Bax JJ, Abraham T, Barold SS, et al. Cardiac resynchronization therapy: Part $1-$ Issues before device implantation. J Am Coll Cardiol 2005;46:2153-67.

4. Chung $\mathbf{E}$, Leon A, Tavazzi $\mathrm{L}$, et al. Results of the predictors of response to CRT (PROSPECT) Trial. Circulation 2008;117:2608-16

5. Lardo AC, Abraham TP, Kass DA. Magnetic resonance imaging assessment of ventricular dyssynchrony: current and emerging concepts. J Am Coll Cardiol 2005;46:2223-8.

6. Chalil S, Stegemann B, Muhyaldeen S, et al. Intraventricular dyssynchrony predicts mortality and morbidity following cardiac resynchronization therapy: a study using cardiovascular magnetic resonance tissue synchronization imaging. J Am Coll Cardiol 2007;50:243-52.

7. White JA, Yee R, Yuan $\mathrm{X}$, et al. Delayed enhancement magnetic resonance imaging predicts response to cardiac resynchronization therapy in patients with intraventricular dyssynchrony. J Am Coll Cardiol 2006;48:1953-60.

8. Chalil S, Stegemann B, Muhyaldeen S, et al. Effect of posterolateral left ventricular scar on mortality and morbidity following cardiac resynchronization therapy. Pacing Clin Elctrophysiol 2007;10:1201-7.

9. Chalil S, Foley P, Muyhaldeen S, et al. Late gadolinium enhancement-cardiovascular magnetic resonance as a predictor of response to cardiac resynchronization therapy in patients with ischaemic cardiomyopathy. Europace 2007;9:1031-7.

10. Aaronson KD, Schwartz JS, Chen TM, et al. Development and prospective validation of a clinical index to predict survival in ambulatory patients referred for cardiac transplant evaluation. Circulation 1997;95:2660-7.

11. Barnett D, Phillips S, Longson C. Cardiac resynchronisation therapy for the treatment of heart failure: NICE technology appraisal guidance. Heart 2007;93:1134-5.

12. Alpert JS, Thygesen K, Antman E, et al. Myocardial infarction redefined-a consensus document of the Joint European Society of Cardiology/American College of Cardiology Committee for the redefinition of myocardial infarction. J Am Coll Cardiol 2000;36:959-69.

13. Assomull RG, Prasad SK, Lyne J, et al. Cardiovascular magnetic resonance, fibrosis, and prognosis in dilated cardiomyopathy. J Am Coll Cardiol 2006;48:1977-85.

14. Myerburg RJ, Castellanos A. Cardiac arrest and sudden cardiac death. In: Braunwald E, ed. Heart disease: a textbook of cardiovascular medicine. New York: WB Saunders, 1997:742-79.

15. Ritter P, Padeletti L, Gillio-Meina L, et al. Determination of the optimal atrioventricular delay in DDD pacing: comparison between echo and peak endocardial acceleration measurements. Europace 1999;1:126-30.

16. Bates DM, Watts DG. Nonlinear regression analysis and its applications. Chichester: Wiley, 1988.
17. Cerqueira MD, Weissman NJ, Dilsizian V, et al. Standardized myocardial segmentation and nomenclature for tomographic imaging of the heart: a statement for healthcare professionals from the Cardiac Imaging Committee of the Council of the American Heart Association. Circulation 2002;105:539-42.

18. Harrell FJ. The Design Package. 2007.

19. Harrell FJ, Lee K, Califf R, et al. Regression modelling strategies for improved prognostic prediction. Stat Med 1984;3:143-52.

20. Harrell FJ, Lee K, Mark D. Multivariate prognostic models: issues in developing models, evaluating assumptions and adequacy, and measuring and reducing errors. Stat Med 1996;15:361-87.

21. Bader H, Garrigue S, Lafitte $S$, et al. Intra-left ventricular electromechanical asynchrony: a new predictor of severe cardiac events in heart failure patients. J Am Coll Cardiol 2004;43:248-56.

22. Fauchier L, Marie 0, Casset-Senon D, et al. Interventricular and intraventricular dyssynchrony in idiopathic dilated cardiomyopathy. A prognostic study with Fourier phase analysis of radionuclide angioscintigraphy. J Am Coll Cardiol 2002;40:2022-30

23. Dries DL, Exner DV, Domanski MJ, et al. The prognostic implications of renal insufficiency in asymptomatic and symptomatic patients with left ventricular systolic dysfunction. J Am Coll Cardiol 2000;35:681-9.

24. Smilde TD, Hillege HL, Navis G, et al. Impaired renal function in patients with ischemic and nonischemic chronic heart failure: association with neurohormonal activation and survival. Am Heart J 2004;148:165-72.

25. Hillege HL, Girbes AR, de Kam PJ, et al. Renal function, neurohormonal activation, and survival in patients with chronic heart failure. Circulation 2000;102:203-10.

26. Lee DS, Austin PC, Rouleau JL, et al. Predicting mortality among patients hospitalized for heart failure: derivation and validation of a clinical model. JAMA 2003;290:2581-7.

27. Anker S, Doehner W, Rauchhaus $\mathrm{M}$, et al. Uric acid and survival in chronic heart failure: validation and application in metabolic, functional, and hemodynamic staging Circulation 2003;107:1991-7.

28. Schoenfeld D. Partial residuals for the proportional hazards regression model. Biometrika 1982;69:239-41.

29. Efron B. Bootstrap method: another look at the jacknife. Ann Stat 1979;7:1-26

30. Tedrow U, Maisel W, Epstein L, et al. Feasibility of adjusting paced left ventricular activation by manipulating stimulus strength. J Am Coll Cardiol 2004;44:2249-51.

31. Breithardt $\mathbf{O A}$, Stellbrink C, Kramer AP, et al. Echocardiographic quantification of left ventricular asynchrony predicts an acute hemodynamic benefit of cardiac resynchronization therapy. J Am Coll Cardiol 2002;40:536-45.

32. Bleeker GB, Kaandorp TAM, Lamb HJ, et al. Effect of posterolateral scar tissue on clinical and echocardiographic improvement after cardiac resynchronization therapy. Circulation 2006;113:969-76.

33. Bax JJ, Bleeker GB, Marwick TH, et al. Left ventricular dyssynchrony predicts response and prognosis after cardiac resynchronization therapy. J Am Col Cardiol 2004; 44:1834-40.

34. Donal E, Tournoux F, Leclercq C, et al. Assessment of longitudinal and radial ventricular dyssynchrony in ischemic and nonischemic chronic systolic heart failure: a two-dimensional echocardiographic speckle-tracking strain study. J Am Soc Echocardiogr 2008;21:58-65.

35. Marcassa C, Campini R, Verna E, et al. Assessment of cardiac asynchrony by radionuclide phase analysis: correlation with ventricular function in patients with narrow or prolonged QRS interval. Eur J Heart Fail 2007;9:484-90.

36. Conca C, Faletra FF, Miyazaki C, et al. Echocardiographic parameters of mechanical synchrony in healthy individuals. Am J Cardiol 2009;103:136-42.

37. Levy WC, Mozaffarian D, Linker DT, et al. The Seattle Heart Failure Model: prediction of survival in heart failure. Circulation 2006;113:1424-33.

38. McCrohon JA, Moon JJC, Prasad SK, et al. Differentiation of heart failure related to dilated cardiomyopathy and coronary artery disease using gadolinium-enhanced cardiovascular magnetic resonance. Circulation 2003;108:54-9.

39. Wyman BT, Hunter WC, Prinzen FW, et al. Mapping propagation of mechanical activation in the paced heart with MRI tagging. Am J Physiol 1999;276:H881-91.

40. Helm RH, Lecquercq C, Faris 0 , et al. Cardiac dyssynchrony analysis using circumferential versus longitudinal strain: Implications for assessing cardiac resynchronization. Circulation 2005;111:2760-7.

41. Westenberg JJM, Lamb H, van der Geest RJ, et al. Assessment of left ventricular dyssynchrony in patients with conduction delay and idiopathic dilated cardiomyopathy. J Am Coll Cardiol 2006;47:2042-8. 